\title{
Características Mecánicas Dinámicas del Fémur Aislado de Perro, Sometido a Prueba de Impacto
}

\author{
Dynamic Mechanical Characteristics of the Isolated Dog Femur, Subjected to Impact Test
}

"Fioretti, C.; "Natali, J.; "Galán, A.; "*Rivera, M. C.; "Moine, R.; ** Varela. P.; "Varela, M.; "***Bonino, F. \& *** Quinteros, R.

FIORETTI, C.; NATALI, J.; GALÁN, A.; RIVERA, M. C.; MOINE, R.; VARELA, P.; VARELA, M.; BONINO, F. \& QUINTEROS, R. Características mecánicas dinámicas del fémur aislado de perro, sometido a prueba de impacto. Int. J. Morphol., 29(3):716-722, 2011.

RESUMEN: El presente trabajo tiene el propósito de brindar información acerca del comportamiento biomecánico del fémur de perro sometido a cargas dinámicas. El objetivo es evaluar la resistencia del fémur frente al impacto, mediante el Método de Ensayo Charpy, relacionando dicha resistencia con las áreas: total, cortical y cavidad medular del hueso, comparándola entre edad y sexo diferentes. Se utilizaron fémur de perro en condiciones aisladas, extraídos postmortem de 30 animales mestizos adultos, provenientes del servicio de zoonosis de la ciudad de Río Cuarto. Se determinó en los fémures derecho e izquierdo: peso y longitud total del hueso, diámetro cráneo-caudal y latero-medial en la mitad de la diáfisis. El fémur izquierdo fue osteotomizado transversalmente en la mitad de su diáfisis. En la superficie de sección se medió espesor de la cortical y diámetro medular. Se obtuvo el área de sección total, cortical y medular. El fémur del miembro pélvico derecho fue sometido al Método de Ensayo Charpy. Los resultados obtenidos fueron analizados estadísticamente encontrándose que el área cortical depende linealmente de la longitud del hueso $(\mathrm{r}=0,62$, p=0,00025); la resistencia del hueso depende de las áreas cortical $(\mathrm{r}=0,71, \mathrm{p}=0,000012)$ y área total (global) $(\mathrm{r}=0,63, \mathrm{p}=0,00021)$ y no del área medular $(\mathrm{r}=0,29, \mathrm{p}=$ 0,12). El análisis exploratorio muestra que no existirían diferencias acerca de la influencia de la edad y el sexo sobre la resistencia del fémur. Tras estos resultados se puede inferir que a medida que aumenta el área cortical aumenta la energía de impacto absorbida.

PALABRAS ClAVE: Perro; Fémur; Prueba de impacto.

\section{INTRODUCCIÓN}

Los huesos son barras componentes del aparato locomotor, sólidas o huecas, sujetas a una moderada tendencia a la curvatura en condiciones de carga excéntrica (Badoux, 1982). Debido a su composición (hidroxiapatita, colágeno y elementos celulares), los huesos antes de romperse experimentan una deformación elástica (reversible) y plástica (irreversible). La resistencia, rigidez y absorción de energía del hueso dependen de sus propiedades materiales (composición, morfología, porosidad), propiedades estructurales (geometría, largo, curvatura) y factores mecánicos (velocidad y orientación de las cargas). Las características estructurales de los huesos varían según la especie, edad y sexo; es por ello que el tejido óseo se remodela de acuerdo a las necesidades funcionales.

El hueso puede adaptarse mecánicamente y modificar sus propiedades materiales y estructurales respondiendo a cargas que se le impongan (Currey, 1984). Las propiedades mecánicas del hueso son modeladas por fuerzas intrínsecas y extrínsecas. Las fuerzas intrínsecas o fisiológicas son estáticas y dinámicas, siendo el resultado del contacto directo de las superficies articulares y ligamentos que rodean a las articulaciones y de las inserciones musculares sobre el hueso. Las fuerzas extrínsecas se originan a partir del ambiente y carecen de limitaciones sobre la magnitud y dirección, por lo cual son potencialmente productoras de fracturas (Schwarz, 1996). La optimización biomecánica es de gran relevancia en la práctica ortopédica veterinaria (Smith, 1985).

La resistencia es importante en la biomecánica del hueso, porque de ella depende el comportamiento del tejido óseo ante una posible fractura, siendo una propiedad intrínseca del hueso, independiente de su forma.

\footnotetext{
* Anatomía Animal, Depto. de Anatomía Animal. Facultad de Agronomía y Veterinaria. Universidad Nacional de Río Cuarto, Argentina.

*** Depto. de Mecánica. Facultad de Ingeniería. Universidad Nacional de Río Cuarto, Argentina.

*** Matemáticas y Bioestadística. Facultad de Agronomía y Veterinaria. Universidad Nacional de Río Cuarto, Argentina.
} 
Para poner a prueba un material (hueso) que resista a un choque o golpe intenso y repentino, debe medirse su resistencia a la ruptura mediante una prueba de impacto o choque. Los ensayos de choque determinan la fragilidad o capacidad de un material de absorber cargas instantáneas. Los ensayos dinámicos de choque se realizan en máquinas denominadas péndulos o martillos pendulares, en las que se verifica el comportamiento de los materiales al ser golpeados por una masa conocida a la que se deja caer desde una altura determinada (González Arias \& Palazon, 1969). Para ello se ha diseñado el Ensayo Charpy, cuyo nombre se debe a su creador, Augustin Georges Albert Charpy.

Esta investigación se fundamenta en la importancia clínica, ortopédica y quirúrgica, que reviste la región del muslo del perro, en la cual las propiedades estructurales del fémur (base ósea de dicha región) determinarán la resistencia frente a fuerzas que actúen sobre él. Por lo tanto las variaciones en la morfología del tejido óseo motivan el análisis de aspectos estructurales, geométricos y biomecánicos del hueso fémur de perro. Dyce et al. (2002) afirman que el fémur es el más fuerte entre los huesos largos del perro. Sin embargo Sánchez-Valverde et al. (1995) considera que dicho hueso es el más afectado por fracturas. Otros reportes indican que la incidencia de fracturas de fémur en pequeños animales representa el $45 \%$ de las fracturas de los huesos largos (Lockett et al., 2004), datos referidos al Hospital de Clínicas de la Universidad Nacional del Nordeste.

El presente trabajo tiene el propósito de brindar información acerca del comportamiento mecánico del fémur de perro sometido a cargas dinámicas.

Los objetivos fueron valuar la resistencia del fémur de perro frente al impacto, mediante el método de ensayo Charpy y, relacionar la resistencia del fémur de perro con la longitud del hueso y con las áreas: total, cortical y de cavidad medular del hueso, comparándola entre diferentes edades y sexo.

\section{MATERIAL Y MÉTODO}

El trabajo se realizó utilizando el hueso fémur de perro en condiciones aisladas. Se emplearon 30 animales mestizos adultos (entre 2 y 10 años) provenientes del servicio de zoonosis de la ciudad de Río Cuarto, Córdoba, Argentina. Dada su procedencia la edad se determinó por cronología dental.

A los perros se les practicó eutanasia utilizando para ello: Acedán, Tiopental sódico, sangrado y fijación general con formol al $3 \%$. Se aislaron por desarticulación ambos fémures y mediante disección convencional se los liberaron de las partes blandas. Los procedimientos citados se llevaron a cabo en la cátedra de Anatomía Veterinaria del Departamento de Anatomía Animal de la Facultad de Agronomía y Veterinaria en la Universidad Nacional de Río Cuarto.

Los animales se agruparon en, grupo A: perros menores a 5 años y grupo B: perros mayor o igual a 5 años. Se determinó en ambos fémur (derecho e izquierdo): peso y longitud total del hueso, diámetro cráneo-caudal y lateromedial en la mitad de la diáfisis.

El fémur izquierdo fue osteotomizado transversalmente en la mitad de su diáfisis, utilizando para ello una sierra manual. En la superficie de sección se midió: espesor de la cortical en los cuadrantes (craneal, caudal, lateral y medial) y diámetro medular (cráneo-caudal y lateromedial) (Fig. 1). Se obtuvieron, mediante fórmula matemática correspondiente, las áreas de sección total (global), área cortical y área medular. Las medidas fueron tomadas mediante el uso de una regla micrométrica de $0,02 \mathrm{~mm}$ de graduación. La elección de lateralidad (derechos o izquierdos) para el hueso osteotomizado responde sólo a un fin metodológico de trabajo. Las medidas del hueso fémur izquierdo serán extrapoladas al fémur derecho contra lateral, utilizando presunción de simetría bilateral (Markel et al., 1994).

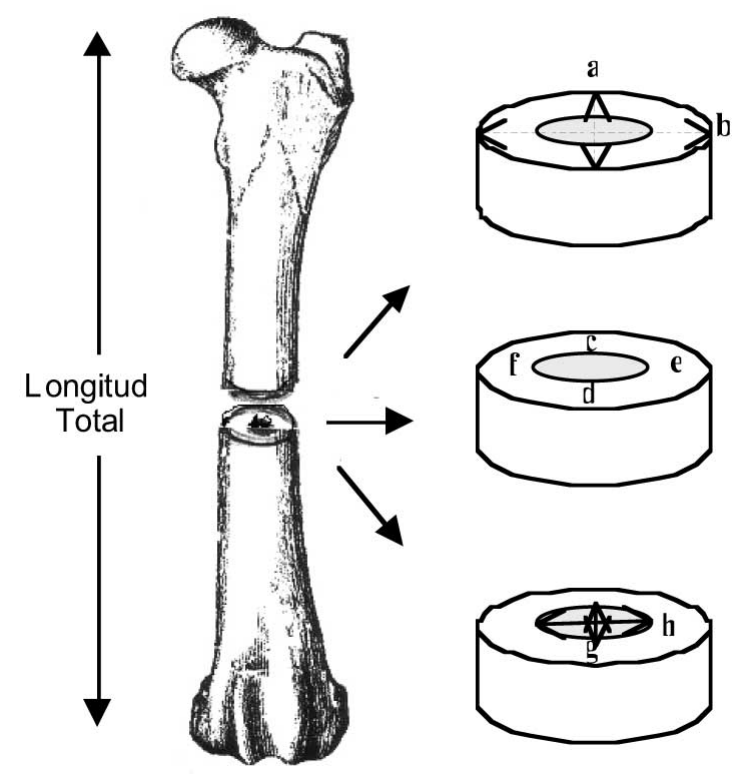

Fig. 1. Vista craneal del fémur izquierdo de perro. a: diámetro cráneo-caudal. b: diámetro latero-medial. Espesor de la cortical en los cuadrantes caudal (c), craneal (d), lateral (e) y medial (f) y diámetro medular cráneo-caudal (g) y latero-medial (h). Tomado y modificado de Evans. 
El hueso fue envuelto en gasa humedecida en solución fisiológica y conservado durante 10 días en freezer a $20 \mathrm{C}^{\circ}$ hasta el ensayo, previamente al mismo se descongelaron los huesos y se llevaron a temperatura ambiente. Reich \& Gefen (2006) demostraron que las propiedades mecánicas del hueso cortical y trabecular no se ven afectadas por la congelación a $-20^{\circ} \mathrm{C}$ hasta un máximo de 100 días.

El fémur del miembro pélvico derecho fue sometido en forma individual a un método dinámico de impacto en dirección transversal en la mitad de la diáfisis. Dicho test de impacto es el Método de Ensayo Charpy a la flexión por impacto y su fundamento consiste en romper de un solo golpe, con un martillo pendular, un material simplemente apoyado en sus extremos. La masa (M), la cual se encuentra acoplada al extremo del péndulo de longitud (L), se deja caer desde una altura $(\mathrm{H})$, mediante la cual se controla la velocidad de aplicación de la carga en el momento del impacto. La energía absorbida (Ea) por el hueso hasta producir su fractura, se determina a través de la diferencia de energía potencial del péndulo antes y después del impacto. Una vez conocido el ángulo inicial de aplicación de la carga (a) y el ángulo final (b) al que se eleva el péndulo después de la rotura completa del hueso, se puede calcular la energía Ea (Ortega, 2006).

El principio de funcionamiento de las máquinas utilizadas es el que ilustra esquemáticamente la Figura 2, en donde una masa o peso $\mathrm{G}$ asegurada a una barra que puede girar libremente sobre un eje $\mathrm{O}$, es elevada a una altura h1, desde su posición vertical de reposo, la que también es posible indicar por el ángulo a1. Si en estas condiciones se la deja caer y en el punto $\mathrm{P}$, ubicado sobre la vertical del desplazamiento del péndulo, se coloca una barra de un material determinado, la masa al chocar con ella producirá su rotura, si la energía que posee el péndulo es mayor que la necesaria para alcanzarla, en cuyo caso continuará su trayectoria elevándose hasta una altura h2 indicada también por el ángulo a2. El trabajo empleado entonces en romper la barra será la diferencia entre la energía inicial del péndulo y la que posee al final de su carrera. (González Arias \& Palazon).

Las pruebas de impacto Charpy se realizan según normas internacionales en las cuales se detallan las dimensiones de las muestras empleadas y la forma de reportar los resultados de los mismos. De acuerdo con las normas ISO, los resultados de los ensayos de impacto se suelen expresar en $\mathrm{kJ} / \mathrm{m} 2$ para lo cual se divide la energía absorbida hasta la fractura del hueso sobre la sección transversal del mismo.

La maquinaria específica para este tipo de ensayo se encuentra en el laboratorio de Mecánica de la Facultad de Ingeniería de la UNRC.

Los datos obtenidos han sido analizados estadísticamente mediante el empleo del paquete estadístico InfoStat versión 2009. Grupo InfoStat, FCA, Universidad Nacional de Córdoba, Argentina. Se realizó en primera instancia una estadística descriptiva tabulando los valores de tendencia central (media) y de dispersión (desvío estándar) para las variables cuantitativas (longitud del hueso, área total (global), cortical y medular) y de frecuencia para las variables cualitativas (edad y sexo).

Así mismo, con fines exploratorios, se hicieron diagramas de dispersión para la resistencia versus el área cortical y de caja para la resistencia versus edad particionado por sexo. Los análisis estadísticos inferenciales consistieron en pruebas de correlación lineal simple y de regresión lineal simple (en caso de ser significativa la correlación a nivel poblacional).

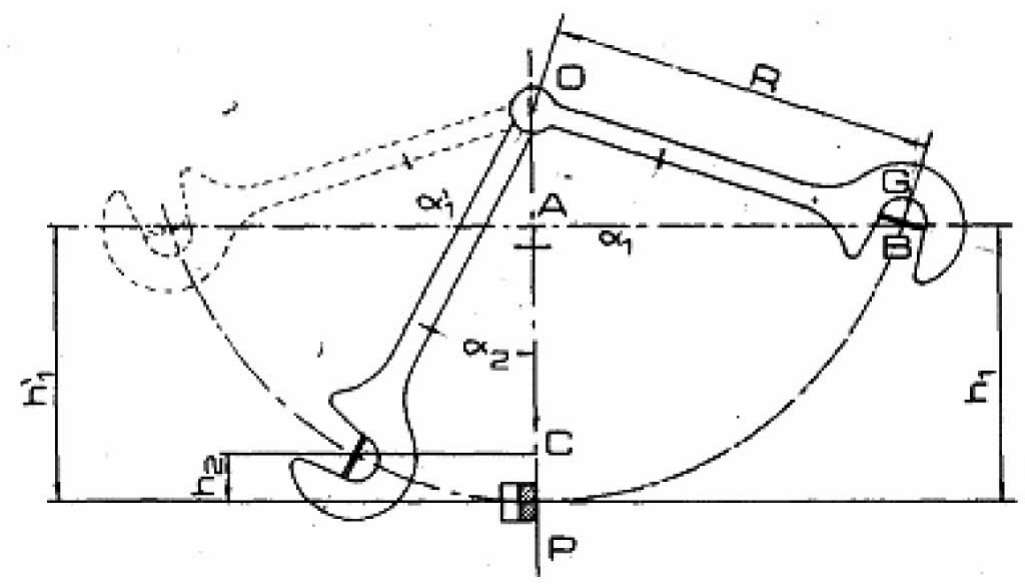

Fig. 2. Esquema del fundamento del Método Charpy. Tomado de González Arias \& Palazon. 


\section{RESULTADOS}

La estadística descriptiva de nuestros datos reflejan (Tabla I), que las variables cuantitativas: longitud fémur izquierdo, área total (global), área cortical, área medular y resistencia presentaron los siguientes promedios, respectivamente: $18,24 \mathrm{~cm}, 1,61 \mathrm{~cm}^{2}, 1,01 \mathrm{~cm}^{2}, 0,60 \mathrm{~cm}^{2}$ y $6,30 \mathrm{~J}$.

En la Figura 3 se observa que la resistencia depende linealmente del área cortical. El análisis exploratorio (Fig. 4: box plot de la resistencia versus edad y sexo) muestra que no existirían diferencias acerca de la influencia de la edad y el sexo sobre la resistencia del fémur, ya que en los grupos A (animales menores a 5 años) y B (animales mayor o igual a 5 años) no se encontraron diferencias estadísticas significativas para dichas variables.

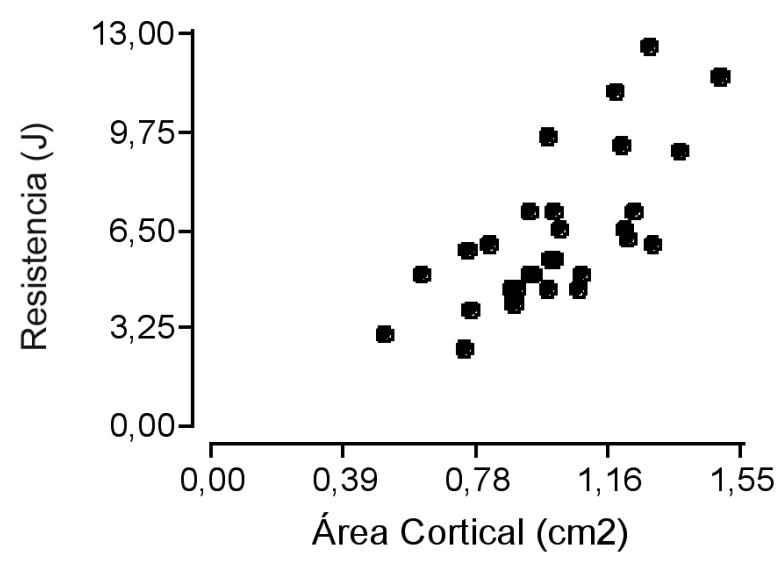

Fig. 3. Comportamiento de la resistencia vs. área cortical.

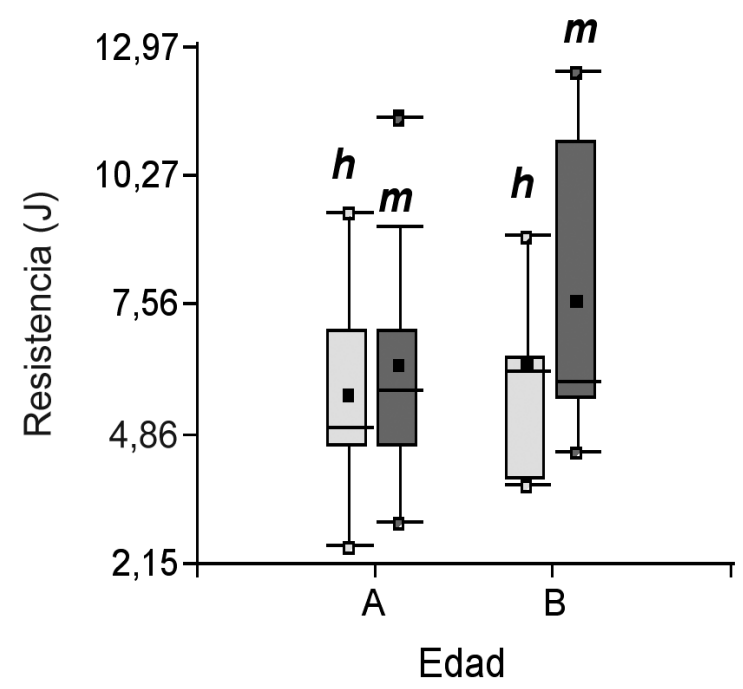

Fig. 4. Comportamiento de la resistencia vs. edad y sexo.
En la estadística inferencial se realizaron análisis de correlación para las variables área cortical versus longitud (Tabla II) y el mismo demostró que el área cortical depende linealmente de la longitud del hueso $(r=0,62, p=0,00025)$. Las Tablas III, IV y V muestran, respectivamente, los análisis de correlación para las variables resistencia versus área cortical; resistencia versus área medular y resistencia versus área total (global). Estos últimos datos nos demuestran que la resistencia del fémur de perro depende de las áreas cortical $(\mathrm{r}=0,71, \mathrm{p}=0,000012)$ y total (global $)(\mathrm{r}=0,63, \mathrm{p}$ $=0,00021)$ y no del área medular $(\mathrm{r}=0,29, \mathrm{p}=0,12)$.

Tabla I. Medidas resumen para variables cuantitativas del fémur izquierdo.

\begin{tabular}{lccc} 
Variable & $\mathrm{n}$ & Media & D. E. \\
\hline Longitud $(\mathrm{cm})$ & 30 & 18,24 & 2,63 \\
Área Total $\left(\mathrm{cm}^{2}\right)$ & 30 & 1,61 & 0,37 \\
Área Cortical $\left(\mathrm{cm}^{2}\right)$ & 30 & 1,01 & 0,23 \\
Área Medular $\left(\mathrm{cm}^{2}\right)$ & 30 & 0,60 & 0,26 \\
Resistencia $(\mathrm{J})$ & 30 & 6,30 & 2,45
\end{tabular}

Tabla II. Correlación de Pearson, fémur izquierdo: coeficientes \ probabilidades.

\begin{tabular}{lcc} 
& Long. $(\mathrm{cm})$ & Área Cortical $\left(\mathrm{cm}^{2}\right)$ \\
Longitud $(\mathrm{cm})$ & 1,00 & $2,5 \mathrm{E}-04$ \\
Área Cortical $\left(\mathrm{cm}^{2}\right)$ & 0,62 & 1,0 \\
\hline
\end{tabular}

Tabla III. Correlación de Pearson, fémur izquierdo: coeficientes \ probabilidades.

\begin{tabular}{lcc} 
& Área Cortical $\left(\mathrm{cm}^{2}\right)$ & Resistencia $(\mathrm{J})$ \\
Área Cortical $\left(\mathrm{cm}^{2}\right)$ & 1,00 & $1,2 \mathrm{E}-05$ \\
Resistencia $(\mathrm{J})$ & 0,71 & 1,0 \\
\hline
\end{tabular}

Tabla IV. Correlación de Pearson, fémur izquierdo: coeficientes \ probabilidades.

\begin{tabular}{lcc} 
& Área Medular $\left(\mathrm{cm}^{2}\right)$ & Resistencia $(\mathrm{J})$ \\
Área Medular $\left(\mathrm{cm}^{2}\right)$ & 1,00 & 0,12 \\
Resistencia $(\mathrm{J})$ & 0,29 & 1,0 \\
\hline
\end{tabular}

Tabla V. Correlación de Pearson, fémur izquierdo: coeficientes \ probabilidades.

\begin{tabular}{lcc} 
& Área Total $\left(\mathrm{cm}^{2}\right)$ & Resistencia $(\mathrm{J})$ \\
Área Total $\left(\mathrm{cm}^{2}\right)$ & 1,00 & $2,1 \mathrm{E}-04$ \\
Resistencia $(\mathrm{J})$ & 0,63 & 1,0 \\
\hline
\end{tabular}




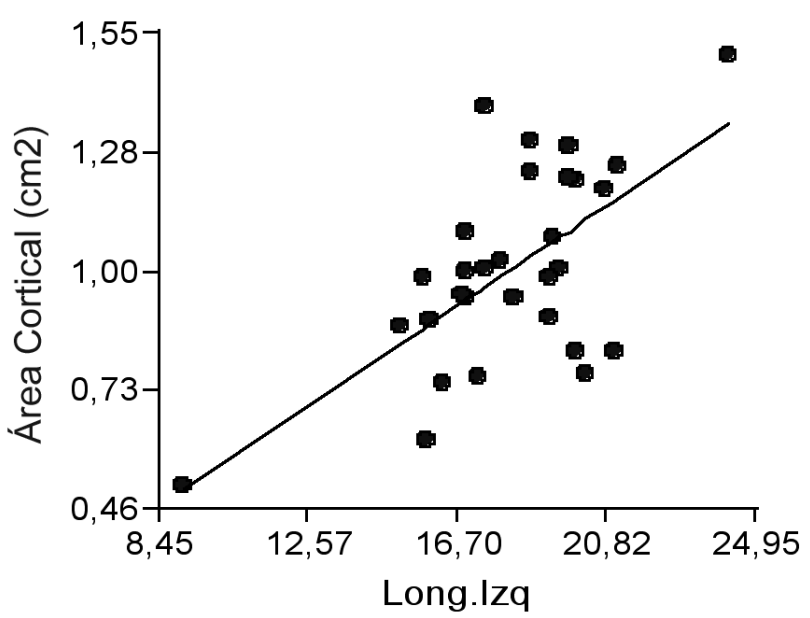

Fig. 5: recta de regresión: área cortical vs. longitud.

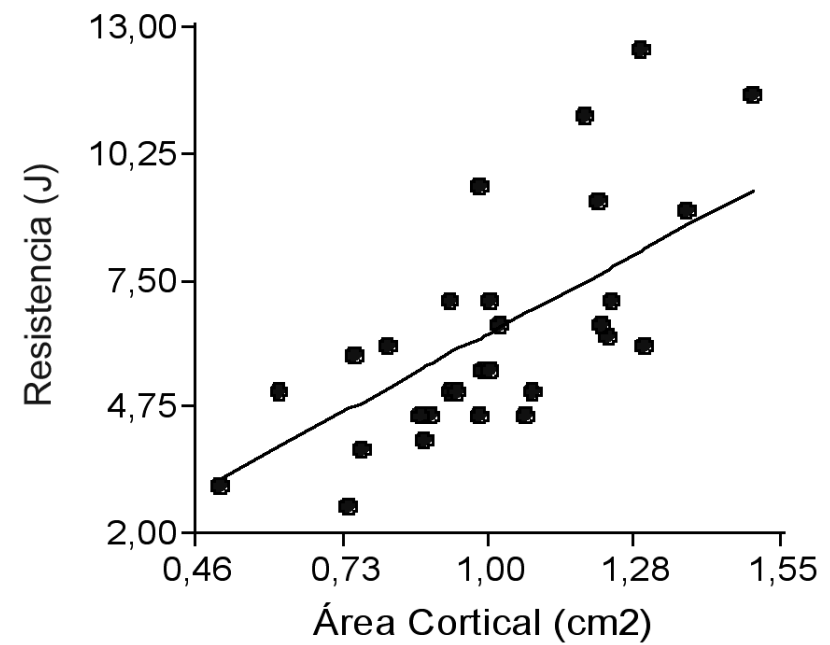

Fig.6: recta de regresión: resistencia vs. área cortical.

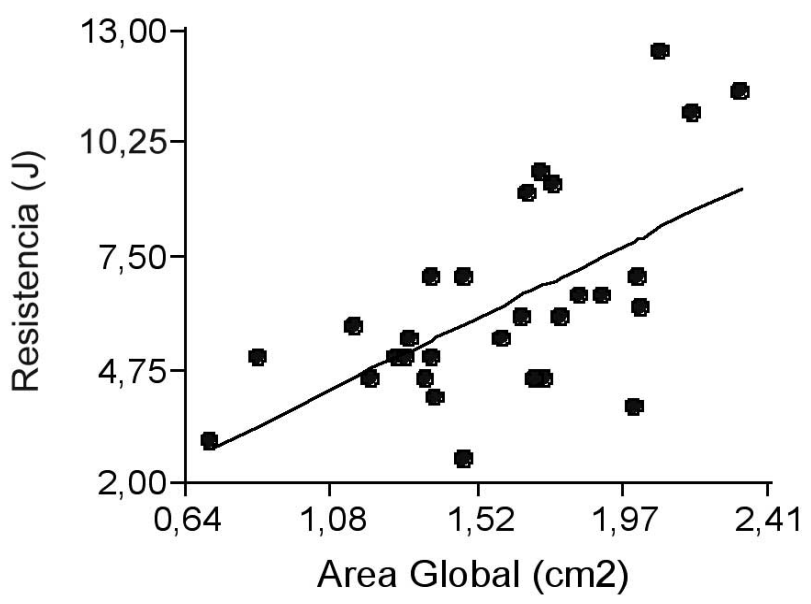

Fig. 7: recta de regresión: resistencia vs. área total.

\section{DISCUSIÓN}

El ensayo mecánico se realizó a temperatura ambiente constante $\left(25^{\circ} \mathrm{C}\right)$ y se utilizó para la totalidad de los ensayos la misma maquinaria de impacto.

Este trabajo determinó que entre la longitud del fémur y el área cortical existe un coeficiente de correlación $\mathrm{r}$ $=0,62$, por lo tanto a medida que la longitud del hueso se incrementa se verifica un aumento del área cortical. Galán et al. (2002) verificó, en el metacarpiano III de potrillos mestizos, que la longitud del hueso tuvo mayor correlación con el área cortical que con el área de cavidad medular la cual se vería influenciada por el aumento en el área cortical. Currey dice que el aumento de la corteza se puede atribuir a la adaptación de las cargas de tensión y compresión, lo cual mejoraría la resistencia del hueso frente al impacto.

Reich \& Gefen experimentan impactos en fémur de pollos y logran determinar que la integridad del hueso trabecular es fundamental para resistir cargas de impacto. Dichos autores concluyen que, en términos biomecánicos, el riesgo de producción de una fractura osteoporótica está determinada por la combinación de factores inherentes a la calidad del hueso (grosor de la cortical, contenido mineral, densidad del hueso trabecular y microarquitectura), como así también por las condiciones de la carga mecánica impuesta. La fuerza requerida para fracturar el hueso es diferente a la resistencia intrínseca por lo que esta carga o fuerza varía con las medidas del hueso (Turner \& Burr, 1993).

La edad avanzada se asocia con una declinación en la resistencia, rigidez ósea y capacidad para absorber energía. En la osteoporosis debido a la pérdida de espesor de la cortical y la microarquitectura trabecular se produce un deterioro y debilitamiento del tejido óseo lo cual impone mayores cargas fisiológicas en el hueso cortical (Reich \& Gefen). Nuestros resultados muestran que el grupo de animales entre 2 y 5 años (A) resistirían menos que el grupo de mayores de 5 años (B), se deberán evaluar otras variables tales como estado nutricional y enfermedades previas para dar un juicio acertado acerca del tema.

Con respecto a las variaciones según el sexo, un ensayo de impacto dinámico en fémur humano realizado por Panagiotopoulos et al. (2005) consideró el sexo y la edad como factores que puede influir en la resistencia ósea a la ruptura. Dentro de los jóvenes el sexo desempeñó un papel significativo, mientras que en el grupo de mayor edad el papel del género fue menos importante. Los huesos fémur de hombres jóvenes son más resistentes a los impactos que los mismos en mujeres jóvenes. El envejecimiento parece 
reducir estas diferencias, por lo cual es interesante conocer que los ancianos tienen la misma absorción de energía de fractura independiente del género (Panagiotopoulos et al.). Nuestros datos no demuestran evidencias estadísticamente significativas para afirmar que en esta muestra existe influencia de la edad y el sexo sobre la resistencia del fémur, si bien se observa en el box plot que los perros machos presentaron una resistencia media mayor que las hembras.

Sumner et al. (1990) realizó comparaciones entre el fémur de perro y el de humano y remarcó que el fémur de perro presenta una "delgada cortical" y por lo tanto una cavidad medular mayor, ocurriendo lo contrario en el fémur humano. Se podría inferir que el fémur de perro presenta una menor resistencia al impacto, condicionado por una menor área cortical.

Tomando como ejemplo un determinado animal (n 28 de nuestra muestra) observamos que con un área cortical de $1,5 \mathrm{~cm}^{2}$ resistió al impacto $11,5 \mathrm{~J}$, en cambio otro animal (n
20) de similar peso, igual talla y sexo, con un área cortical de $0,51 \mathrm{~cm}^{2}$ resistió al impacto 3J. Natali et al. (2008) dice que "los huesos en los cuales prevalece el tamaño del área de sección cortical, responden con una mayor resistencia ante la compresión axial, en cambio en los huesos en los cuales prevalece el área de sección total, no necesariamente responden con una mayor resistencia". En nuestro trabajo queda demostrada la importancia del tamaño del área cortical respecto de la resistencia de los huesos en condiciones aisladas.

La resistencia del fémur en nuestro trabajo, presentó un coeficiente de correlación muestral $\mathrm{r}=0,71$ respecto al área cortical y de 0,63 respecto al área total (global). El coeficiente de correlación muestral entre resistencia y área de cavidad medular fue de 0,29. Tras estos resultados se puede inferir entonces que a medida que aumenta el área cortical aumenta la energía de impacto absorbida. En cambio, un aumento en el área de cavidad medular no necesariamente implica una mayor cantidad de energía absorbida ya que éstas variables no se correlacionan.

FIORETTI, C.; NATALI, J.; GALÁN, A.; RIVERA, M. C.; MOINE, R.; VARELA, P.; VARELA, M.; BONINO, F. \& QUINTEROS, R. Dynamic mechanical characteristics of the isolated dog femur, under impact test. Int. J. Morphol., 29(3):716-722, 2011.

SUMMARY: This paper is intended to provide information about biomechanical behavior femur at impact using the Charpy test method relating the resistance in the following areas: total, cortical and medullary bone cavity, comparing these between different ages and sexes. Dog femors were used in isolated conditions, removed postmortem from 30 adult animals, defined raceless, from the zoonoses service of the city of Rio Cuarto. Weight, total bone length, cranio-caudal diameter and lateral medial half of the diaphysis, were determined in the left and right femur. The left femur was osteotomized transversely in the middle of the diaphysis. In the sectional area thickness of the cortical and medullary diameters were measured. We obtained the total sectional area, cortical and medullary. The femur of the right lower limb was subjected to the Charpy test method. Results were statistically analyzed and found that cortical area depends linearly on the length of the bone $(\mathrm{r}=0.62, \mathrm{p}=0.00025)$, bone strength depends on the cortical areas $(\mathrm{r}=0.71, \mathrm{p}=0.00012)$ and total (global) areas ( $\mathrm{r}=0.63$, $\mathrm{p}=0.00021)$ and not of the medullary area $(\mathrm{r}=0.29, \mathrm{p}=0.12)$. Exploratory analysis showed that no difference existed regarding age and sex influence on the strength of the femur. Following these results we can infer that as cortical area increases, the impact energy absorbed also increases.

KEY WORDS: Dog; Femur; Impact Test.

\section{REFERENCIAS BIBLIOGRÁFICAS}

Badoux, D. M. Bioestática y biomecánica. En: Getty, R. (Ed.). Anatomía de los Animales Domésticos. 5ta ed. Barcelona, Salvat, 1982. pp. 55-95. Tomo I.

Currey, J. D. The mechanical propertiesof materials and the estructure of bone. The Mechanical Adaptation of Bone. Princeton, University Press, 1984. pp.3-37.

Dyce, K. M.; Sack, W. O. \& Wensing. C. J. G. Anatomía Veterinaria. $2^{\mathrm{a}}$ ed. Buenos Aires, Mc Graw-Hill Interamericana, 2002. pp.94-105.

Galán, A.; Rivera, M. C.; Moine, R.; Ferraris, G.; Gigena,
M. S. \& Natali, J. Propiedades morfométricas del metacarpiano III de potrillos mestizos. Rev. Chil. Anat., 20(3):285-90, 2002.

Gonzalez Arias, A. \& Palazon, A. Ensayos industriales de materiales, combustibles y lubricantes. Buenos Aires, Ediciones Litenia, 1969. pp.211-6.

Lockett, M. B. Incidencia De fracturas de huesos largos en caninos atendidos en el Hospital de Clínicas, Facultad Ciencias Veterinarias- UNNE, en el período 1995-2004. XXV Sesión de Comunicaciones Científicas 2004. Fac. de Cs. Veterinarias Universidad Nacional del Nordeste, 2004. 
FIORETTI, C.; NATALI, J.; GALÁN, A.; RIVERA, M. C.; MOINE, R.; VARELA, P.; VARELA, M.; BONINO, F. \& QUINTEROS, R. Características mecánicas dinámicas del fémur aislado de perro, sometido a prueba de impacto. Int. J. Morphol., 29(3):716-722, 2011.

Natali, J.; Wheeler, J. T.; Kohl, R. \& Varela, P. Comparación de las características mecánicas estáticas del fémur aislado de perro, con y sin la colocación de una placa de ortopedia fabricada en polipropileno. Int. J. Morphol., 26(4):791-7, 2008.

Markel, M. D.; Sielman, E.; Rapoff, A. J. \& Kohles, S. S. Mechanical properties of long bones in dogs. Am. J. Vet. Res., 55(8):1178-83, 1994.

Ortega, Y. Prueba de impacto: ensayo Charpy. Rev. Mex. Fis., 52(1):51-7, 2006.

Panagiotopoulos, E.; Kostopoulos, V.; Tsantzalis, S.; Fortis, A. P. \& Doulalas, A. Impact energy absorption by specimens from the upper end of the human femur. Injury, 36(5):613-7, 2005.

Reich, T. \& Gefen, A. Efecto de la pérdida de hueso trabecular en la velocidad de deformación cortical durante el impacto en un modelo in vitro de fémur aviar. Biomed. Eng. Online, 5:45-45, 2006.

Sanchez-Valverde, M. A.; Tovar, M. C.; Agut, A.; Laredo, F. G. \& Murciano, J. Estudio clínico de las fracturas pediátricas en la especie canina. Arch. Med. Vet., 27(2):55-67, 1995.

Schwarz, P. D. Biomecánica de las fracturas del esqueleto apendicular: causas y valoración. In: Bojrab, M. J. Fisiopatología y clínica quirúrgica en animales pequeños. Buenos Aires, Editorial Intermédica, 1996. pp.1040-58.

Smith, G. K. Biomechanics Pertinent to Fracture Etiology, Reduction, and Fixation. In: Newton, CH. A.; Nunamaker, D. M. \& Lippincott, C. Textbook of Small Animal Orthopaedics. Philadelphia, Lippincott, 1985. pp.195-230.

Sumner, D. R. J.; Devlin, T. C.; Winkelman, D. \& Turner, T. $\mathrm{M}$. The geometry of the adult canine proximal femur. $J$. Orthop. Res., 8(5):671-7, 1990.

Turner, C. R. \& Burr, D. B. Basic biomechanical measurements of bone: a tutorial. Bone, 14(4):595-608, 1993.
Dirección para correspondencia:

José Natalí

Anatomía Animal

Departamento de Anatomía Animal

Facultad de Agronomía y Veterinaria

Universidad Nacional de Rio Cuarto

Campus Universitario. (5800)

Rio Cuarto, Cba.

ARGENTINA

Teléfono 54-358-4676419

Fax 54-358-4680280

Email: jnatali@uncr.edu.ar

Recibido : 06-01-2011

Aceptado: 16-05-2011 